\title{
Modeling of turbine wheel assemblies taking into account the deformation of parts
}

\author{
Mariya V. Yanyukina ${ }^{1, *}$, and Mikhail A. Bolotov ${ }^{1}$ \\ ${ }^{1}$ Samara University, Engine Production Technology Department, 34, Moskovskoye shosse, Samara, \\ 443086, Russia
}

\begin{abstract}
The article researches an impeller. The assembly process requires to accurately adhere to the given assembly parameters. To do this, preliminary calculations are carried out, they must take into account a number of factors, such as tolerances in processing the parts to be assembled, the tensile-compression stiffness, torsional rigidity, and others. The article studies the influence of blades deformation with the contact pressure on the accuracy of the goods assembly parameters.
\end{abstract}

\section{Introduction}

Goods performance depends on the precision of separate parts production, as well as on the quality of the assembly process. The goods assembling process is characterized by high labor intensity, complexity and is often performed manually. The accuracy of the assembly process is highly influenced by the assemblies made, characterized by the correctness of their formation and the assignment of the dimensionally accurate parameters of the constituent links. To enhance the accuracy of the assembly links calculation, which affects the goods assembly accuracy in the process of technological preparation, it is necessary to clarify and detail the calculated models by accounting for the existing factors. The measured links models are based on the basic concepts and methods of probability theory and mathematical statistics. The components of the links, called the links of the dimensional chain, are dimensional quantities that have tolerances. The real geometry of the parts differs from the nominal geometry. For this reason, dimensional calculations must take into account the probabilities of dimensional parameters, determined by the technological processes of manufacturing parts within the tolerance. Given the relatively low rigidity and high requirements for the accuracy of the assembly parameters, it is especially important to take into account the influence of the power factor in the process of calculating the assembly chains.

The existing publications pay much attention to the issue of modeling assembly links.

Paper [1] speaks about three stages of assembly of any aviation structure, characterized by the formation of gaps, their elimination by pressing, the appearance of internal stresses and, as a consequence, deformations of parts. Timely corrections, as the author points out, require forecasting the quality indicators of the assembled goods based on the initial parameters of the parts proceed to the assembly.

\footnotetext{
*Corresponding author: mary392@mail.ru
} 
The authors of article [2] suggest considering linear graph models, which allow describing several assembly circuits and establishing their mutual influence. According to the authors, "this approach makes it possible to simultaneously consider more than one dimensional circuit, taking into account the interference of circuits, and to use a model for automating the calculation methods of assembly dimensional chains." However, the paper does not show the influence of the power factor.

A.V. Mukholzoev's paper [3] discusses the automated calculation of dimensional chains, prefers the theory of graphs, but points out that "the graph of dimensional chains does not contain information about the mutual arrangement of surfaces."

V.L. Solomakho and Yu. B. Spesivtseva in their paper [4] consider a complex technique for calculating dimensional chains that will allow increasing the accuracy of less "expensive" links and reducing the accuracy of more "expensive" ones, so that the cost of goods production is minimal if the required operational parameters are ensured. To determine the optimized tolerances of links in a dimensional chain, the authors propose using the maximum-minimum method, as well as the probabilistic one.

The national research in the field of dimensional analysis of the parameters of the assembly process shows that up to now the problem has been worked out in most cases for a simple statement of the problem. The works of foreign authors are aimed to a greater extent at the preliminary process of assembly in CAD systems, subject to modeling of parts with production tolerances $[5,6,7]$.

The present paper considers a model for calculating the assembly chains of the impeller, taking into account the deformation of parts.

\section{Model}

Let us examine an assembly unit of a turbine disk, including a disk with banded blades. The most significant requirements are imposed on the accuracy of the following assembly parameters: the gap between the shrouds of adjacent $C_{b}$ blades, the gap between the locking flanges of adjacent blades $C_{i}$ and the tension between the butt surfaces of the shrouds of the blades $\mathrm{I}_{\mathrm{b}}$. In addition, it is necessary to take into account the gaps between the shank tips and the grooves of the disk, which are necessary to compensate for temperature deformations (Fig. 1).

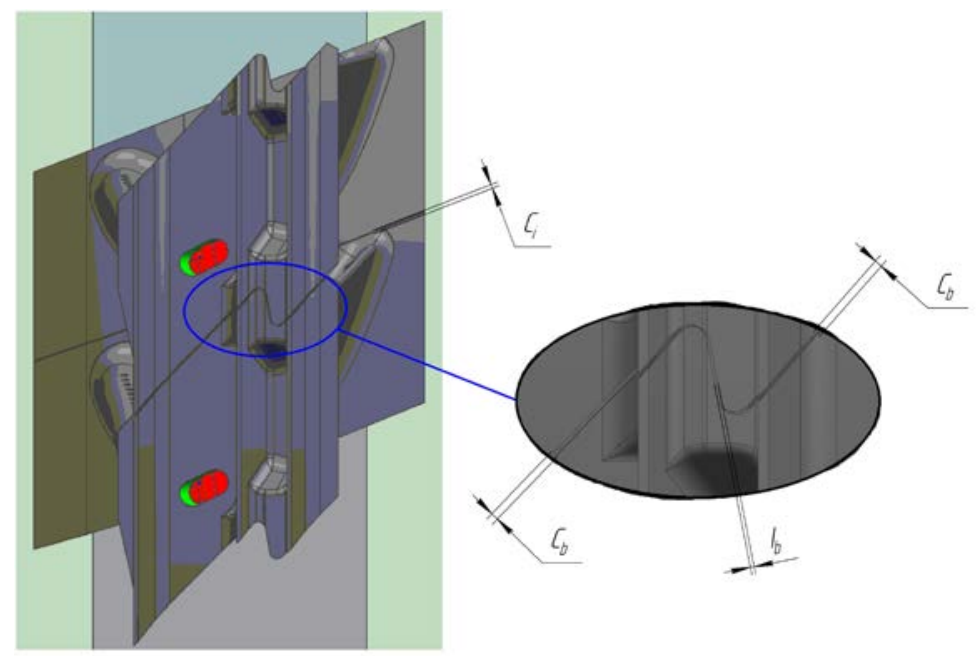

Fig. 1. The main parameters, to which special requirements are imposed when assembling the impeller. 
The blade in the groove of the disc can move in two directions (Fig. 1): the movement in the plane ZoX (the so-called "rolling") and XoY (turning the blade around its axis). When rolling the blades on the same sides of the fir-tree root, either an interference or a gap that exceeds the allowable value. If turning the blade on the sides of the lock with the same name, there is both a gap and interference on the different end surfaces of the disk. The cause of the turn of the blade around the axis can be the impact of the force of contact pressure between the shrouds exceeding the permissible value. The contact pressure is determined by the amount of interference on the contact surfaces of the shrouds of adjacent blades (Fig. 2).

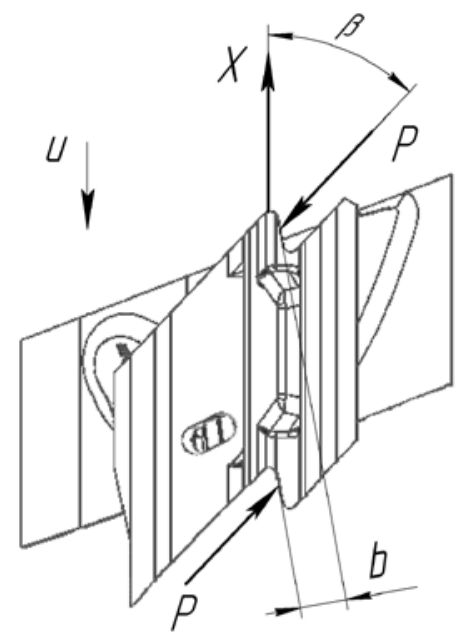

Fig. 2. The force of the contact pressure acting on the blades of the impeller.

According to article [8] to ensure the tightness set by the designer on the shrouds, the following ratio must be satisfied:

$$
\begin{gathered}
t_{p} \cos \beta=b \\
\Delta b=b-b_{0}=\frac{P}{c_{1}} \\
\Delta \beta=\beta-\beta_{0}=-\frac{P t_{p} \sin \beta}{c_{2}}
\end{gathered}
$$

$t_{p}=2 \pi r_{u} / z-$ blade pitch at the location of the platform,

$\mathrm{b}_{0}$ and $\mathrm{b}$ - distance between contact surfaces, respectively, before and after assembly,

$\beta_{0}$ and $\beta-$ bevel of contact surfaces to the circumferential direction before and after assembly,

$\mathrm{P}$ - force of contact pressure between tip shrouds,

$\mathrm{c}_{1}$ - the rigidity of the tip shroud for compression (including crushing over contact surfaces),

$\mathrm{c}_{2}$ - torsion stiffness of blade.

The value $\Delta \beta$ indicates to what extent the blade turns, provided the applied force $\mathrm{P}$, while considering the blade as a non-rigid body.

This parameter can be calculated in two ways [8]: 


$$
\begin{gathered}
\Delta \beta=-\frac{\left(P t_{p} \sin \beta_{0}\right) / c_{2}}{1+\left(P t_{p} \cos \beta_{0}\right) / c_{2}} \\
\Delta \beta \approx \frac{\cos \beta_{0}-\cos \beta}{\sin \beta_{0}}=-\frac{b_{0}-t_{p} \cos \beta_{0}}{t_{p} \sin \beta_{0}}
\end{gathered}
$$

We use the formula (4) to determine the bevel directly through the force on the contact surfaces. In this case, we calculate the force as:

$$
P \approx \frac{b_{0}-t_{p} \cos \beta_{0}}{\frac{1}{c_{1}}+\frac{t_{p}}{c_{2}}\left(t_{p}-b_{0} \cos \beta_{0}\right)}
$$

Having data on the geometry of the blade prior to assembly, with the chosen force $\mathrm{P}$, we can determine the change in the bevel of the contact surface of the tip shroud to the circumferential direction, as well as the distance between the contact surfaces after assembly.

\section{Results and discussion}

According to the calculations based on the design documentation, the following values of bevel the contact surfaces to the circumferential direction and the distance between the contact surfaces were obtained: $\Delta \beta$ approximately 1 degree, and $\Delta \mathrm{b}=1.9 \mathrm{~mm}$, that is, for each contact spot of the shrouds is $\sim 0,8 \mathrm{~mm}$.

The previous study [9] was devoted to the computer calculation of dimensional chains in order to determine the amount of interference between the contact surfaces of the shrouds. The paper considered the blade as a rigid body, that is, the deformations of the tip shrouds were not taken into account as a result of the action of the contact pressure force. The calculation was carried out by statistical modeling methods and consisted in solving the dimensional circuits of the impeller during assembly. As a result, the distance between the contact surfaces after the assembly was equal to $4.35 \mathrm{~mm}$. If the deformation of the blade is taken into account, this value is $3.72 \mathrm{~mm}$.

Comparing the results obtained in the course of the two studies, it is clear how important the consideration of the stiffness of the blade, its ability to deform under load is.

\section{Conclusion}

The results of study showed that it is necessary to take into account not only the geometric, but also the physical characteristics of the parts. Calculating part parameters taking into account the assumptions simplifies the tasks, but at the same time introduces appreciable tolerances $[10,11]$.

When modeling the assembly links of aviation profile parts, the deformation of the constituent parts is of great importance. The paper aimed at using the example of an impeller, the deformations of the shroud platforms were taken into account in the calculations. However, not all influencing factors were presented in this abstract.

The shroud size can vary (increase) under the influence of heating the blades. This also has an effect on the contact force of the pressure and, as a consequence, on the tension and turn of the blade in the lock. 
This work was supported by the Ministry of Education and Science of the Russian Federation in the framework of the implementation of the Program "Research and development on priority directions of scientific-technological complex of Russia for 2014-2020

\section{References}

1. Yu.N. Zolotarev, The designing of statistical model for extrapolation the quality of the aviation construction in assembly, Bulletin of Voronezh state technical University, v. 1(10), (2014)

2. M.G. Galkin, A.S. Smagin, The Practice of Dimensional Modelling of Assembly Operations Using Stationary Compensators, News of higher educational institutions. Engineering, v. 12(669), pp. 71-76 (2015)

3. A.V. Muholzoev, The algorithm for automated calculation of technological dimensional chains, Bulletin of the South Ural State University. Ser. Mechanical Engineering Industry, v. 3(15), pp. 48-55 (2015)

4. V.L. Solomaho, Yu. B. Spesivtseva, The integrated approach to the calculation of dimensional chains, Bulletin of Polotsk State University, v. 8, pp. 63-70 (2009)

5. I. Jbira, M. Tlija, B. Louhichi, A. Tahan, CAD/Tolerancing integration: Mechanical assembly with form defects, Advances in Engineering Software, v. 114, pp. 312-324 (2017)

6. Zuowei Zhua, Lihong Qiaoa, Nabil Anwerb, An Improved Tolerance Analysis Method Based on Skin Model Shapes of Planar Parts, Procedia CIRP, v. 56, pp. 237-242 (2016)

7. Katia Lupinetti, Franca Giannini, Marina Monti, Jean-Philippe Pernot, Multi-criteria retrieval of CAD assembly models, Journal of Computational Design and Engineering, v. 5, pp. 41-53 (2018)

8. I.A. Birger, B.F. Shorr, G.B. Iosilevich, Calculation of the strength of machine parts (Engineering, Moscow, 1993)

9. M.V. Yanyukina, M.A. Bolotov, N.V. Ruzanov, Interrelated Dimensional Chains in Predicting Accuracy of Turbine Wheel Assembly Parameters, IOP Conf. Series: Materials Science and Engineering, v. 327, (2018) 